POS PROCEEDINGS

\title{
Performance and aging of OPERA bakelite RPCs
}

\author{
A. Paoloni* \\ INFN Laboratori Nazionali di Frascati, Italy \\ E-mail: alessandro.paolonielnf.infn.it
}

\section{A. Bertolin, R. Brugnera, F. Dal Corso, S. Dusini, A. Garfagnini, L. Stanco}

Padua University and INFN, Padua, Italy

\section{A. Candela, M. D’Incecco, M. Lindozzi}

INFN Laboratori Nazionali del Gran Sasso, Assergi, Italy

\section{A. Cazes}

IPNL, Université Claude Bernard, Lyon 1, France

G. Corradi, G. Felici, A. Longhin, A. Mengucci, G. Papalino, M. Spinetti, M. Ventura, L. Votano

INFN Laboratori Nazionali di Frascati, Italy

\section{B. Klicek, M. Stipcevic}

Rudjer Boskovic Institute (IRB), Zagreb, Croatia

\begin{abstract}
OPERA is an experiment dedicated to the observation of $v_{\mu}$ into $v_{\tau}$ oscillations through $\tau$ appearance on the CNGS beam.

The experiment is composed by two identical super-modules, each with a target section (made of emulsion/lead bricks alternated to a scintillator Target Tracker) and a muon spectromter (instrumented with bakelite electrodes RPCs and drift tubes).

The OPERA RPC system is composed of about 1000 RPCs for an instrumented area of $3000 \mathrm{~m}^{2}$. The RPCs are operated in streamer mode and flushed with the gas mixture $\mathrm{Ar} / \mathrm{C}_{2} \mathrm{H}_{2} \mathrm{~F}_{4} / i-\mathrm{C}_{4} \mathrm{H}_{10} / \mathrm{SF}_{6}=75.4 / 20.0 / 4.0 / 0.6$ at five refills/day in open flow.

The present performance of the RPC system after six years of operation are presented. The aging
\end{abstract} status of the detector is also described.

XI workshop on Resistive Plate Chambers and Related Detectors - RCP2012,

February 5-10, 2012

INFN Laboratori Nazionali di Frascati Italy

\footnotetext{
*Speaker.
} 


\section{Introduction}

OPERA is an experiment dedicated to the observation of $v_{\mu}$ into $v_{\tau}$ oscillations through $\tau$ appearance on the CNGS (CERN Neutrino to Gran Sasso) beam [1]. Nuclear emulsion layers, alternated to $1 \mathrm{~mm}$ thick lead sheets, are packed into "bricks", to ensure a vertex reconstruction at the $\mu \mathrm{m}$ level [2].

Inside the two target sections of the experiment, the bricks are arranged in walls, which are alternated to target tracker layers made of crossed scintillator strips. Each target section is followed by a muon spectrometer, to improve the charm background rejection. Each spectrometer is composed by an iron dipole magnet with drift tubes as precision trackers. Resistive plate chambers are used for triggering the drift tubes and for tracking inside the magnetized iron [3].

The detector operation started on 2006, when the first CNGS neutrino events were registered by the electronic detectors [4]. Since 2008 all bricks have been inserted: the OPERA target is made of about 150000 bricks corresponding to a target mass of $1.25 \mathrm{kton}$. The complete analysis of 2008 and 2009 data samples, corresponding to $5.3 \times 10^{19}$ proton-on-target, resulted in the observation of only one tau candidate [5], consistent with the expectation of $(1.65 \pm 0.41)$ events. The estimated number of background events is $(0.16 \pm 0.03)$, corresponding to a significance of $95 \%$. The analysis of 2010 and 2011 data samples is in progress.

A picture of the OPERA experiment is shown in figure 1.

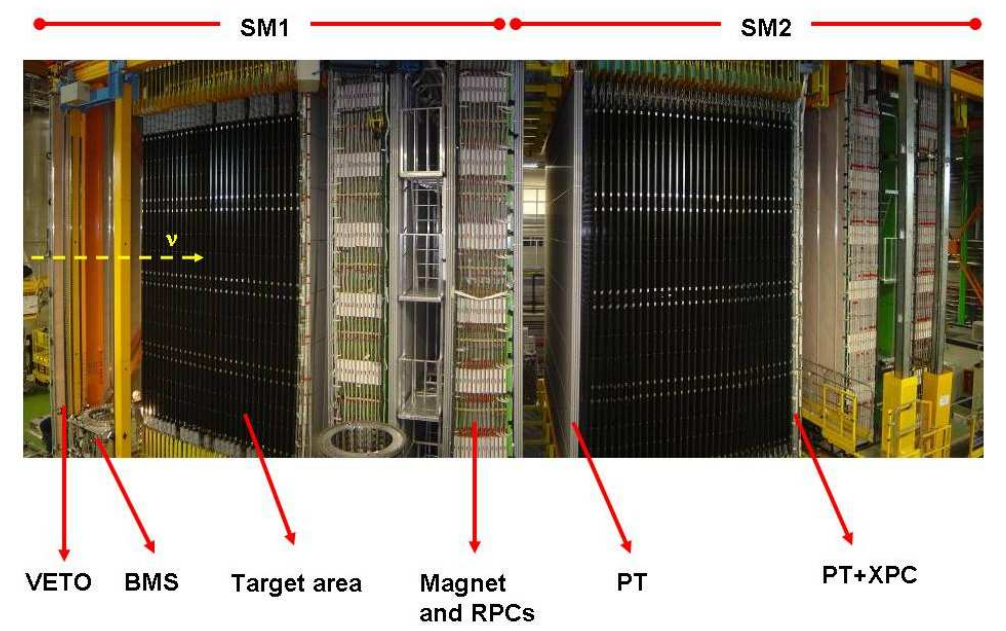

Figure 1: A fish-eye view of the OPERA experiment. The upper red horizontal lines indicate the position of the two identical supermodules (SM1 and SM2). The "target area" is made up of planes of walls filled with lead-emulsion bricks interleaved with planes of plastic scintillators (TT): the black covers visible in the photograph are the end-caps of the TT. Arrows show also the position of the VETO planes, the drift tubes (PT) followed by the XPC, the magnets and the RPC installed among the magnet slabs. The Brick Manipulator System (BMS) is also visible. The direction of incoming neutrinos from CERN is indicated by the yellow arrow. 


\section{The RPC system}

Resistive Plate Chambers with bakelite electrodes are displaced into 22 layers in each spectrometer, inserted into $2 \mathrm{~cm}$ gaps inside the magnetized iron. One RPC layer is made of 21 detectors, covering an area of about $64 \mathrm{~m}^{2}$, arranged into three columns and seven rows. One row is the basic unit for the high voltage, the operating current monitoring and the gas distribution system. The RPC layers are read-out by means of orthogonal strips, whose pitch is $2.6 \mathrm{~cm}$ for the vertical strips (measuring the bending coordinate) and $3.5 \mathrm{~cm}$ for the horizontal ones.

Two additional layers are placed in each Super-Module between the Target Tracker and the spectrometer; they are called XPCs because of the $\pm 43^{\circ}$ inclined read-out strips for solving tracking ambiguities.

Given the low counting rate in the underground Gran Sasso laboratory, $\sim 20 \mathrm{~Hz} / \mathrm{m}^{2}$, bakelite plates with resistivity $\rho>5 \times 10^{11} \Omega \mathrm{cm}$ have been used as electrodes. OPERA RPCs are operated in streamer and flushed with the gas mixture $\mathrm{Ar} / \mathrm{C}_{2} \mathrm{H}_{2} F_{4} / i-\mathrm{C}_{4} \mathrm{H}_{10} / S F_{6}=75.4 / 20.0 / 4.0 / 0.6$ at five refills/day. The voltage applied across the $2 \mathrm{~mm}$ gas gap is $5.7 \mathrm{kV}$ at an environment pressure of about $900 \mathrm{mbar}$ and a temperature ranging between 15 and $18^{\circ} \mathrm{C}$.

The read-out strips are terminated on one side on their characteristic impedance, while on the other side are connected to the front-end electronics through twisted flat cables with lengths going from 6.5 to $15 \mathrm{~m}$. Given the large amplitude streamer signals, no amplification is needed. Signals from the vertical strips, measuring the bending coordinate, are discriminated at $40 \mathrm{mV}$ on $110 \Omega$, while the threshold for the horizontal strips has been lowered at the beginning of 2009 run from its initial value of $40 \mathrm{mV}$ to $26 \mathrm{mV}$ on $110 \Omega$, in order to correct for the different impedance matching with the long twisted flat cables connecting the read-out strips to the discriminators.

The XPC layers and 7 out of 22 RPC layers in each spectrometer are instrumented with dedicated Timing Boards (TBs) for triggering the drift tubes. The Timing Boards are high impedance discriminators installed in the read-out strip panels and operated at $20 \mathrm{mV}$ threshold on $110 \Omega$.

A complete description of the OPERA RPC system, including front-end electronics and data acquisition, can be found in [2].

\section{Performance and aging}

The two OPERA spectrometers have been operated during the 2011 CNGS run for about 9 months, from March to November. The downtime of the system has been about $0.1 \%$ due to gas system failures.

In figure 2 the RPC layer efficiencies measured on cosmic rays are shown, from 2009 to 2011. The principal contribution is $4 \%$ geometric acceptance, while RPC layers with values lower than $90 \%$ are typically affected by high voltage failures in one or more rows, fixed at the end of the run.

The main aging effect observed on OPERA bakelite RPCs is the progressive drying of the electrodes. Indeed the detectors are flushed with a dry gas mixture as they share the same gas mixing unit with the VETO glass RPCs. In figure 3 it is shown the evolution of the efficiency (corrected for the geometric acceptance) as a function of the position along the gas flow (1=entrance, $3=$ =xit). A moderate efficiency decrease, between 1 and $2 \%$, maximum at the gas entrance, is observed. It 

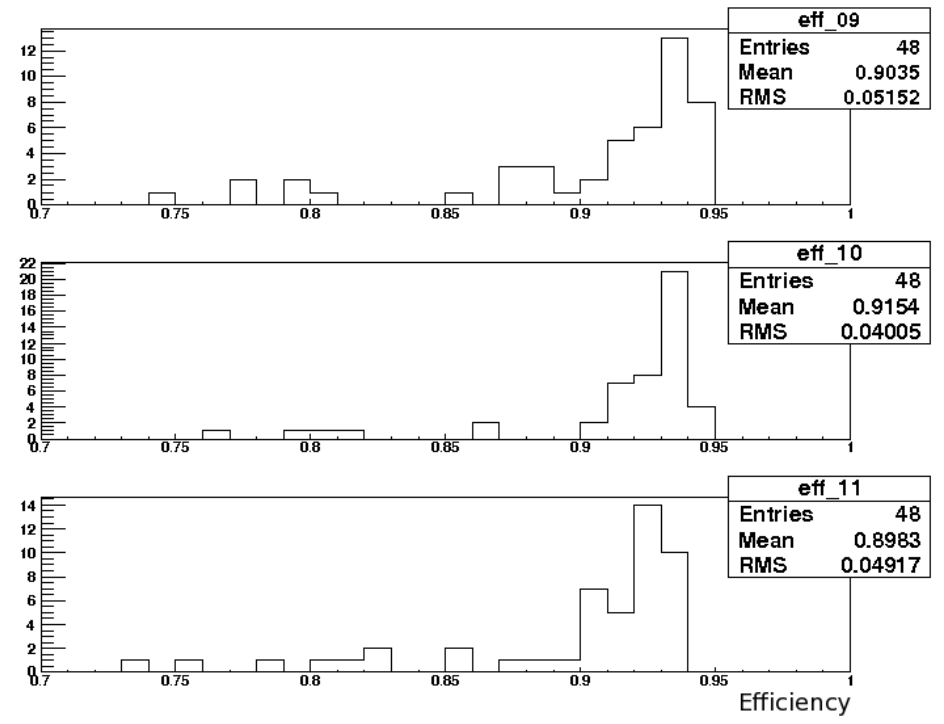

Figure 2: RPC and XPC layer efficiencies measured on cosmic rays on 2009 (upper plot), 2010 (central plot) and 2011 (lower plot) runs.

is worth mentioning that typical currents are below $500 \mathrm{nA}$ for single rows and that only $3 \%$ of the system shows currents above $4 \mu \mathrm{A}$.

The effect of the progressive electrode drying, corresponding to a lower effective voltage across the gas gap, can be seen also on the cluster size, whose time evolution is reported in figure 4.

The OPERA Timing Boards have been used to estimate the RPC time resolution considering

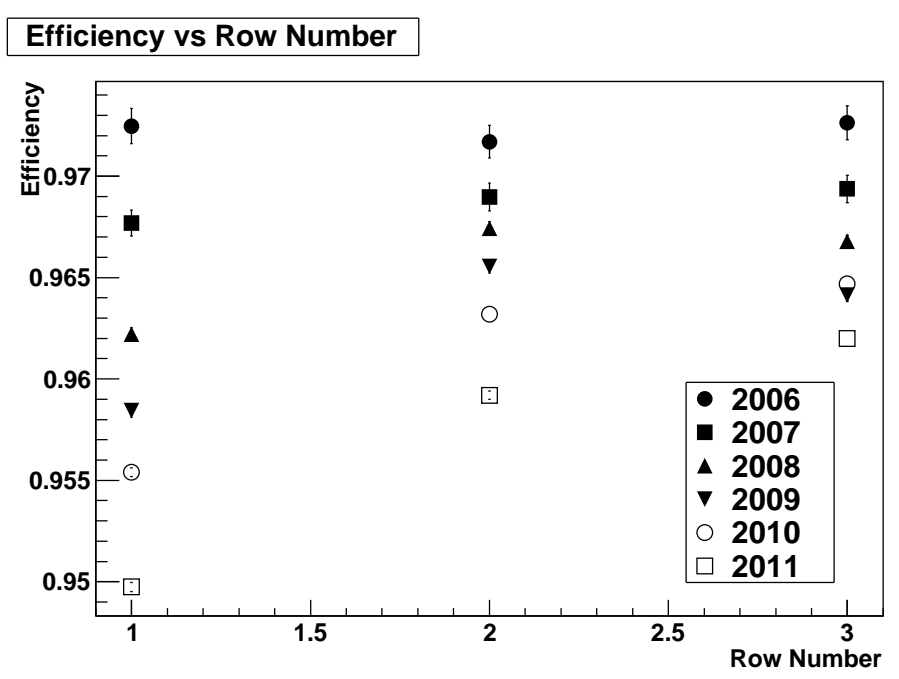

Figure 3: Average efficiency (corrected by the geometric acceptance) as a function of the RPC position along the gas flow (1=entrace, $3=$ exit) for the different years of data taking. 


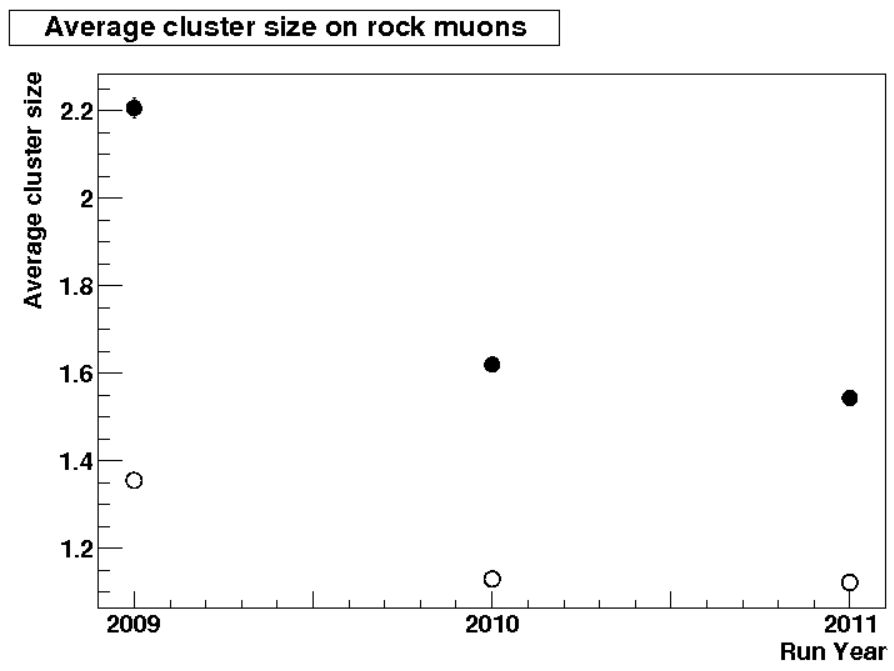

Figure 4: RPC average cluster sizes measured on CNGS neutrino-produced muons for the vertical (empty circles) and horizontal (full circles) read-out strips.

the time-of-flight between consecutive rows: the distribution is fitted with a gaussian and the corresponding sigma is then divided by $\sqrt{2}$. In figure 5 the time resolution values measured from 2009 to 2011 are shown.

\section{Conclusions}

The OPERA RPC system completed its sixth year of operation. The only observed aging effect is a moderate increase of the bakelite electrodes resistivity due to flushing with a dry gas mixture.

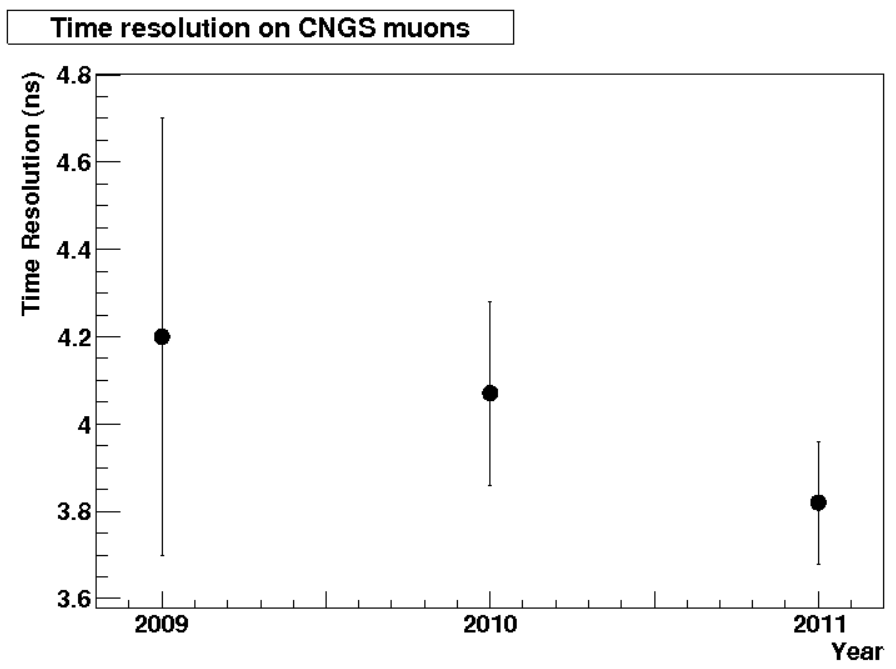

Figure 5: RPC time resolutions measured in the last three years of data taking on rock muons.. 
The effect is monitored and the detector performance is still within the specifications, with layer efficiencies above $90 \%$ and time resolutions below $5 \mathrm{~ns}$.

\section{References}

[1] M. Guler et al., OPERA proposal, CERN/SPSC 2000-028, SPSC/P318, LNGS P25/2000.

[2] R. Acquafredda et al., JINST 4, P04018 (2009).

[3] M. Ambrosio et al., IEEE Transactions on Nuclear Science, vol.51, NO.3, (JUNE 2004) 975-979.

[4] R. Acquafredda et al., New Journal of Physics, 8 (2006) 303.

[5] The OPERA Collaboration, New Journal of Physics 14 (2012) 033017. 